F. CAMPANA

Une version géométrique généralisée du théorème du produit de Nadel

Bulletin de la S. M. F., tome 119, no 4 (1991), p. 479-493

<http://www.numdam.org/item?id=BSMF_1991_119_4_479_0>

(C) Bulletin de la S. M. F., 1991, tous droits réservés.

L'accès aux archives de la revue «Bulletin de la S. M. F. » (http: //smf.emath.fr/Publications/Bulletin/Presentation.html) implique l'accord avec les conditions générales d'utilisation (http:/www.numdam.org/ conditions). Toute utilisation commerciale ou impression systématique est constitutive d'une infraction pénale. Toute copie ou impression de ce fichier doit contenir la présente mention de copyright.

\title{
Numdam
}

Article numérisé dans le cadre du programme

Numérisation de documents anciens mathématiques

http://www.numdam.org/ 
Bull. Soc. math. France,

119,1991 , p. $479-493$.

\section{UNE VERSION GÉOMÉTRIQUE GÉNÉRALISÉE DU THÉORÈME DU PRODUIT DE NADEL}

PAR

F. CAMPANA $(*)$

RÉsumé. - On montre que le lieu de non-intégrabilité locale $S$ d'un diviseur ample $L$ d'une variété projective complexe lisse $X$ contient toute courbe immergée $C$ suffisamment mobile de $X$ qui rencontre $S$, pourvu que le degré normalisé de $C$ relatif à $L$ soit assez petit. On en déduit, en particulier, la finitude du nombre de familles de déformation des variétés de Fano de dimension fixée telles que $b_{2}=1$.

ABSTRACT. - We show that the non-integrability locus $S$ of an ample divisor $L$ of a smooth complex projective variety $X$ contains every sufficiently movable immersed curve $C$ of $X$ which meets $S$, provided the normalized degree of $C$ relatively to $L$ is sufficiently small. From this follows in particular the finiteness of the number of deformation families of Fano manifolds of given dimension with $b_{2}=1$.

\section{Introduction}

Dans [N], où A. NADEL démontre que $c_{1}(X)^{4} \leq 20^{4}$ si $X$ est une variété de Fano de dimension 4 avec $b_{2}(X)=1$, l'ingrédient essentiel de la démonstration est le théorème suivant :

Si $\Delta^{m}$ est un polydisque de $\mathbb{C}^{m}$, et si $\varphi$ est une fonction presque plurisousharmonique sur $M=\Delta^{m} \times \mathbb{P}_{1}(\mathbb{C})$ telle que : $\int_{c} i \partial \bar{\partial} \varphi<1$ pour toute courbe $C \cong \mathbb{P}_{1}(\mathbb{C})$, fibre de la projection naturelle $\pi: M \rightarrow \Delta^{m}$, alors le sous-ensemble analytique fermé $S(\varphi)$ de $M$ constitué de points au voisinage desquels $\varphi$ n'est pas localement intégrable est saturé pour $\pi$ c'est-à-dire que $S(\varphi)=\pi^{*} \pi_{*} S(\varphi)$.

Sa démonstration repose sur des techniques élaborées de théorie $L^{2}$ de Hörmander et utilise l'identité de Bochner-Kodaira-Nakano.

Nous montrons ici que ce résultat reste vrai lorsque $\pi: M \rightarrow \Delta^{m}$ est une application analytique surjective propre et lisse à fibres connexes de

(*) Texte reçu le 23 janvier 1991.

F. Campana, Département de Mathématiques, Université de Nancy I, B.P. 219, 54506

Vandœuvre-les-Nancy Cedex, France.

BULLETIN DE LA SOCIÉTÉ MATHÉMATIQUE DE FRANCE 0037-9484/1991/479/\$5.00

(C) Société mathématique de France 
dimension un, mais de genre arbitraire, pourvu que l'on se limite à la version géométrique du théorème du produit, où $\varphi$ est une puissance (négative) de la norme d'une section analytique d'un fibré en droites hermitien sur $M$. La démonstration, directe, est extrêment simple (mais utilise de manière essentielle le fait, dû à H. HIRONAKA, qu'une modification propre est localement dominée par une suite finie d'éclatements de centres lisses). On en déduit alors, comme dans $[\mathrm{N}]$, un théorème de confinement (voir $\S 4$ ). Les conséquences géométriques sont obtenues à l'aide de la stratégie introduite dans $[\mathrm{N}]$.

Par des méthodes géométriques analogues à celles de [C], on déduit du théorème de confinement le résultat principal suivant :

Théorème. - Soit $X$ une variété projective lisse et connexe de dimension $n, L$ un fibré en droites ample sur $X$ et $C$ une courbe libre immergée de $X$ (voir définitions 3.2 ) telle que:

$$
\left(L^{n}\right)^{1 / n} \geq n \cdot(L \cdot C)
$$

où $L^{n}$ est le nombre d'intersection de L. Alors $X$ contient un diviseur effectif $E$ tel que

$$
E \cdot C=0 .
$$

Utilisant alors le fait (voir [N]) que la théorie de Mori fournit des courbes rationnelles libres immergées de degré $d(X) \leq(n+1)$ si $X$ est de Fano, on obtient le :

Corollaire. - Si X est, de plus, une variété de Fano (i.e. $c_{1}(X)$ est ample) telle que $b_{2}(X)=1$, on $a$ :

$$
c_{1}(X)^{n} \leq(n \cdot d(X))^{n} \leq(n(n+1))^{n} .
$$

D'où résulte la finitude du nombre des familles de déformation de variétés de Fano de dimension $n$ avec $b_{2}=1$. ([K-M])

Ce résultat a été établi, avec une démontration de nature algébrogéométrique dans $\left.\left[\mathrm{N}^{\prime}\right]\right)$. Une démonstration reposant sur des méthodes de géométrie différentielle est proposée dans $[\mathrm{T}]$.

\section{Intégrabilité locale de diviseurs}

1.0 Conventions. - Soit $M$ une variété analytique complexe connexe de dimension $m \geq 1$. On note $\Delta^{m}$ un polydisque de $\mathbb{C}^{m}, D$ un diviseur de $M$ défini par $D=\sum_{i \in I} m_{i} D_{i}$, où $D_{i}$ est un diviseur effectif irréductible

$$
\text { TOME } 119-1991-\mathrm{N}^{\circ} 4
$$


réduit défini en $p \in M$ par une équation locale $f_{i}=0$ s'annulant en $p$ à l'ordre $\mu_{i}$ et où $m_{i} \in \mathbb{R}$, avec $I$ ensemble fini.

On note $\mu_{p}(D)$ la multiplicité en $p$ de $D$, définie par

$$
\mu_{p}(D)=\sum_{I} \mu_{i} m_{i}
$$

On dit que $D$ est intégrable en $p$ si la fonction :

$$
g:=\left(\prod_{i \in I}\left|f_{i}\right|^{-2 m_{i}}\right)
$$

est localement intégrable sur un voisinage de $p$ dans $M$.

On notera $S(D)$ l'ensemble des points de $M$ en lesquels $D$ n'est pas intégrable.

On dira que $D$ est à croisements normaux en $p$ s'il existe des coordonnées locales $\left(z_{1}, \ldots, z_{m}\right)$ centrées en $p$ et telles que $f_{i}=z_{i}$ pour $i \in I \subset\{1, \ldots, m\}$.

On a alors les résultats bien connus suivants (voir exemple [D] que cette présentation reproduit) :

Proposition 1.1. - On suppose $D$ effectif, c'est-à-dire $: m_{i} \geq 0$ pour tout $i \in I$. Alors :

1) $p \in S(D)$ si $\mu_{p}(D) \geq m$.

2) $p \notin S(D)$ si $\mu_{p}(D)<1$.

3) Si $D$ est à croisements normaux en $p$, alors $p \notin S(D)$ si et seulement si : $m_{i}<1$ pour tout $i \in I$.

Démonstration. - On choisit des coordonnées locales $\left(z_{i}\right)_{i \leq m}=z$ centrées en $p$ et on pose :

$$
r(z)=r=\left(\sum_{i=1}^{n}\left|z_{i}\right|^{2}\right)^{1 / 2} .
$$

1) On a $g(z) \geq \prod_{i \in I} r^{-2 m_{i} \mu_{i}} \geq r^{-2 \mu_{p}(D)} \geq r^{-2 m}$. Donc $g$ n'est pas $L_{\text {loc }}^{1}$ en $p$ et $p \in S(D)$ si $\mu_{p}(D) \geq m$.

2) On a $g(z)=\prod_{i \in I}\left|z_{i}\right|^{-2 m_{i}}$ est intégrable si $m_{i} \leq 1$ pour tout $i \in I$ ), et si $D$ est à croisements normaux en $p$.

3) On peut se ramener au cas où les $m_{i}$ sont rationnels de même dénominateur $m_{i}=p_{i} / q \geq 0$.

Alors $g(z)=|f(z)|^{-2 / q}$, où $f(z)=\prod_{i \in I} f_{i}(z)^{p_{i}}$ est analytique.

Le théorème de préparation de Weierstrass permet alors de supposer que $f(z)$ est un polynôme de Weierstrass $f(z)=z_{1}^{r}+a_{i}(\tilde{z}) z_{1}^{r-1}+\cdots+a_{r}(\tilde{z})$, 
où $r:=\sum_{i \in I} p_{i} \mu_{i}=q \mu_{p}(D)$, où $\tilde{z}=\left(z_{2}, \ldots, z_{m}\right)$ et où les $a_{j}$ sont analytiques au voisinage de $\tilde{z}=0$ et $a_{j}(0)=0$ pour $j \leq r$.

On peut supposer que si $f(z)=0$ alors $\left|z_{1}\right| \leq 1$, lorsque $\|\tilde{z}\| \leq \nu$. Soient alors, pour $\tilde{z}_{0}$ fixé, $b_{1}\left(\tilde{z}_{0}\right), \ldots, b_{r}\left(\tilde{z}_{0}\right)$ les solutions de $f(z)=0$ pour $z=\left(z_{1}, \tilde{z}_{0}\right)$.

L'inégalité de la moyenne arithmético-géométrique fournit l'inégalité, si $z=\left(z_{1}, \tilde{z}_{0}\right)$ :

$$
|f(z)|^{-2 / q}=\left|\prod_{j=1}^{r}\left(z_{1}-b_{j}\left(\tilde{z}_{0}\right)\right)\right|^{-2 / q} \leq \frac{1}{r} \sum_{j=1}^{r}\left|z_{1}-b_{j}\left(\tilde{z}_{0}\right)\right|^{-2 r / q}
$$

et donc :

$$
\begin{aligned}
\int_{\left|z_{1}\right| \leq 1} g\left(z_{1}, \tilde{z}_{0}\right) \mathrm{d} z_{1} \wedge \mathrm{d} \bar{z}_{1} & \leq \frac{i}{r} \sum_{j=1}^{r} \int_{\left|z_{i}\right| \leq 1}\left|z_{1}-b_{j}\left(\tilde{z}_{0}\right)\right|^{-2 r / q} \mathrm{~d} z_{1} \wedge \mathrm{d} \bar{z}_{1} \\
& \leq i \int_{\left|z_{1}\right| \leq 2}\left|z_{1}\right|^{-2 r / q} \mathrm{~d} z_{1} \wedge \mathrm{d} \bar{z}_{1}
\end{aligned}
$$

Puisque $r / q<1$, l'intégrale de droite converge. La conclusion résulte alors du théorème de Fubini.

Proposition 1.2. - Soit $V$ une sous-variété analytique complexe fermée de $M$ de codimension $c \geq 2$. Soit $\varepsilon: \widetilde{M} \rightarrow M$ l'éclatement de $M$ le long de $V$ et $E=\varepsilon^{-1}(V)$ le diviseur exceptionnel de cet éclatement. Soit $\widetilde{D}:=\varepsilon^{*}(D)-(c-1) E$. Alors $: S(D)=\varepsilon_{*}(S(\widetilde{D}))$.

On dit que le couple $(\widetilde{M}, \widetilde{D})$ est déduit de $(M, D)$ par l'éclatement de $V$. Démonstration. - Soit Jac $\left(\varepsilon\left(z^{\prime}\right)\right)$ le jacobien de l'application $\varepsilon$ au point $z^{\prime} \in \widetilde{M}$ : cette fonction s'annule à l'ordre $(c-1)$ le long de $E$ et nulle part ailleurs, puisque $K_{\widetilde{M}}=\varepsilon^{*} K_{m}+(c-1) E$, où $K_{M}$ est le fibré canonique de $M$.

D'autre part, si $U$ est un voisinage de $p \in M$, on a :

$$
i^{m} \int g(z) \mathrm{d} z \mathrm{~d} \bar{z}=i^{m} \int_{\varepsilon^{-1}(u)} \varepsilon^{*} g\left(z^{\prime}\right)\left|\operatorname{Jac}\left(\varepsilon\left(z^{\prime}\right)\right)\right|^{2} \mathrm{~d} z^{\prime} \mathrm{d} \bar{z}^{\prime} .
$$

(Ceci peut s'écrire plus rigoureusement à l'aide d'une partition de l'unité.)

On voit donc que $p \in S(D)$ si et seulement si il existe $p^{\prime} \in S(\widetilde{D})$ tel que $\varepsilon\left(p^{\prime}\right)=p$, puisque le diviseur de $\operatorname{Jac}\left(\varepsilon\left(z^{\prime}\right)\right)$ est $(c-1) E$. 
Corollaire. $-S(D)$ est un sous-ensemble analytique fermé de $M$.

Démonstration. - Il suffit, d'après la Proposition 1.2 et le théorème de projection de Remmert, de montrer ceci pour un couple $\left(M^{\prime}, D^{\prime}\right)$ déduit de $(M, D)$ par un nombre fini d'éclatements de sous-variétés.

Un théorème d'Hironaka permet alors de se ramener au cas où $D^{\prime}$ est à croisements normaux en chacun de ses points. La conclusion résulte alors de la Proposition $1.1 ; 3$.

\section{Le théorème de saturation}

THÉORÈme 2.1. - Soit $\pi: M \rightarrow \Delta^{m-1}$ un morphisme propre et lisse (à fibres connexes, donc) et $D=D_{0}+\pi^{*}(\Delta)$, dans lequel :

1) $D_{0}$ est effectif et chacune de ses composantes irréductibles est génériquement finie sur $\Delta^{m-1}$. Soit $d$ le degré de $D_{0}$, c'est-à-dire $d=D \cdot C$, où $C$ est une fibre de $\pi$.

2) $\Delta$ est un diviseur de $\Delta^{m-1}$ (pas nécessairement effectif). Alors, si $d<1, S(D)=\pi^{*} \pi_{*} S(D)$.

Démonstration. - Soit $\nu: \widetilde{\Delta}^{m-1} \rightarrow \Delta^{m-1}$ l'éclatement d'une sousvariété $W$ de codimension $c \geq 2$ de $\Delta^{m-1}$; soit

$$
\tilde{\pi}: \widetilde{M}:=M \times_{\Delta^{m-1}} \widetilde{\Delta}^{m-1} \rightarrow \widetilde{\Delta}^{m-1}
$$

le morphisme déduit de $\pi$ par le changement de base $\nu$ et $\varepsilon: \widetilde{M} \rightarrow M$ la projection naturelle : c'est aussi l'éclatement de $V:=\pi^{-1}(W)$ dans $M$. Soit $H:=\nu^{-1}(W)$ (resp. $E$ ) le diviseur exceptionnel de $\nu$ (resp. $\varepsilon$ ). On a $E=\tilde{\pi}^{*}(H)$.

Soit $(\widetilde{M}, \widetilde{D})$ le couple déduit de $(M, D)$ par $\varepsilon$. D'après la ProposiTION 2, on a :

$$
\varepsilon_{*}(S(\widetilde{M}, \widetilde{D}))=S(M, D) .
$$

Puisque :

$$
\widetilde{D}=\varepsilon^{*}(D)-(c-1) E=\varepsilon^{*}(D)-(c-1) \tilde{\pi}^{*} H,
$$

on a :

1) les conditions d'effectivité de $D$ énoncées dans le THÉORÈme 4 sont satisfaites,

2) le degré de $\widetilde{D}$ est encore $d<1$.

Donc si $\tilde{\pi}^{*} \tilde{\pi}_{*} S(\widetilde{D})$, on a aussi $S(D)=\tilde{\pi}^{*} \tilde{\pi}_{*}(S(D))$. Il suffit donc d'établir le résultat lorsque $\left(M^{\prime}, D^{\prime}\right)$ est déduit de $(M, D)$ par un nombre fini d'éclatements dans $\Delta^{m-1}$. Le théorème d'aplatissement d'Hironaka permet alors de supposer que $D=D_{0}+\pi^{*}(\Delta)$, où $\pi: D_{0} \rightarrow \Delta^{m-1}$ est une application finie en tout point de $\left|D_{0}\right|$. 
Lemme 2.2. - Dans la situation du Théorème 2.1, supposons que $D_{0}$ soit fini sur $\Delta^{m-1}$. Alors : $S(D)=\pi^{*} S(\Delta)$.

Démonstration. - Soient $p \in M$ et $z=\left(z_{1}, \tilde{z}\right) \in \mathbb{C} \times \Delta^{m-1}$ des coordonnées locales dans lesquelles $\pi$ est la projection linéaire sur $\Delta^{m-1}$. On décompose $I=J \cup K$, où $J$ (resp. $K$ ) indexe les composantes irréductibles de $D_{0}$ (resp. $\Delta$ ).

Soient :

$$
g_{0}:=\prod_{i \in J}\left|f_{i}\right|^{-2 m_{i}} \quad \text { et } \quad g_{1}:=\prod_{i \in K}\left|f_{i}\right|^{-2 m_{i}}=\pi^{*} h=\prod_{i \in K}\left|\pi^{*} h_{i}\right|^{-2 m_{i}}
$$

où les composantes de $\Delta$ sont définies par les équations $h_{i}$ sur $\Delta^{m-1}$.

Donc $g=g_{0} \pi^{*} h$. La démonstration de l'assertion 2 de la ProposiTION 1 montre que pour tout $\tilde{z}_{0} \in \Delta^{m-1}$, la fonction $g_{0}\left(z_{1}, \tilde{z}_{0}\right)=\varphi_{0}\left(z_{1}\right)$ est intégrable et son intégrale est bornée par celle d'une fonction intégrable fixe. Il résulte alors du théorème de Fubini que $g$ est intégrable au voisinage de $p$ si $h$ est intégrable au voisinage de $\pi(p)$.

Inversement, si $h$ n'est pas intégrable au voisinage de $\pi(p), g$ n'est pas intégrable au voisinage des points $p^{\prime}$ de $\pi^{-1} \pi(p)$ qui ne sont pas sur $D_{0}$ et a fortiori, pas aux points de $\left(\pi^{-1} \pi(p) \cap D_{0}\right)$; donc $g$ n'est intégrable en aucun point de $\pi^{-1} \pi(p)$.

REMARQUE 2.3. - Un examen de la démonstration permet de constater que le théorème d'aplatissement n'a pas été utilisé, mais seulement le fait qu'une modification propre est localement dominée par une suite finie d'éclatements de centres lisses.

\section{Courbes libres}

3.0. Notations. - On désignera toujours dans la suite par $X$ une variété projective lisse et connexe; par $n$ sa dimension complexe, par $L \in \operatorname{Pic}(X)$ un fibré en droites ample sur $X$; par $\rho(X)$ le nombre de Picard de $X$, c'est-à-dire le rang de son groupe de Picard; par $C$ une courbe effective de $X ; \operatorname{par} \mathcal{C}(X)$ la variété de Chow de $X ; \operatorname{par}\{\mathcal{C}\}$ le point de $\mathcal{C}(X)$ correspondant à $C$ (ses composantes irréductibles étant munies des multiplicités adéquates); par $T$ une sous-variété projective irréductible de $\mathcal{C}(X) ;$ par $\left(C_{t}\right)_{t \in T}$ la famille universelle de cycles algébriques de $T$ paramétrée par $T$; par $G \subset T \times X$ le graphe de la relation d'incidence définie par $T$ (c'est-à-dire $G=\left\{(t, x) \in T \times X\right.$ tels que $\left.x \in C_{t}\right\}$ ). On notera enfin :

$$
p: G \longrightarrow T \quad \text { et } \quad q: G \longrightarrow X
$$

TOME $119-1991-\mathrm{N}^{\circ} 4$ 
les restrictions des projections naturelles de $T \times X$. On notera $\tilde{\nu}: \widehat{G} \rightarrow G$ la normalisation de $G$, et $\tilde{p}:=p \circ \tilde{\nu}$ et $\tilde{q}:=q \circ \tilde{\nu}$ les composées de $\tilde{\nu}$ avec $p$ et $q$. Si $Y$ est une sous-variété projective de $X$, on notera $\operatorname{codim}_{X}(Y)$ sa codimension dans $X$.

Définition 3.1. - On note $d_{L}(C):=(L \cdot C) /\left(L^{n}\right)^{1 / n}$ le $L$ degré normalisé de $C$. On remarquera qu'il est inchangé si l'on remplace $L$ $\operatorname{par}(k \cdot L)$, avec $k$ entier positif. Si $\rho(X)=1$, on notera simplement $d(C)$ ce degré.

\section{DÉFINITION 3.2.}

1. On dira que $C$ est immergée si $C$ est réduite, irréductible et si $(i \circ \nu): \widehat{C} \rightarrow X$ est une immersion, où $i$ est l'injection de $C$ dans $X$ et $\nu: \widehat{C} \rightarrow C$ la normalisation de $C$.

2. On dira que $C$ est $T$-libre si $T$ est une composante irréductible de $\mathcal{C}(X)$ contenant $\{C\}=t_{0}$ telle que $q: G \rightarrow X$ soit ouverte en tout point de $\left\{t_{0}\right\} \times C=p^{-1}\left(t_{0}\right)$. On dira que $C$ est libre s'il existe $T$ telle que $C$ soit $T$-libre. On dira que $C$ est strictement $T$-libre si $T$ est lisse en $t_{0}$, si $C$ est irréductible et réduite, si $\widehat{G}$ est lisse et si $\tilde{q}$ est ouverte en tout point de $\tilde{\nu}^{-1}\left(\left\{t_{0}\right\} \times C\right)$, et submersive en son point générique.

EXEMPLE 3.3. - Si $C \subset X$ est une courbe rationnelle réduite et irréductible et si $(i \circ \nu)^{*}\left(T_{X}\right)$ est engendré par ses sections globales (c'està-dire de la forme $\bigoplus_{i=1}^{n} \mathcal{O}_{C}\left(a_{i}\right)$, avec $a_{i} \geq 0$ pour tout $i$ ), alors $C$ est strictement libre, d'après $[\mathrm{K}]$. Si de plus, $h^{1}\left(\widehat{C},(i \circ \nu)^{*}\left(T_{X}\right)(-2)\right) \leq 1$ (c'est-à-dire $a_{1}=2$ et $a_{i} \in\{0,1\}$ pour $i \geq 2$ ), alors $C$ est immergée. Ici, $T_{X}$ est le fibré tangent à $X$.

Proposition 3.4. - Soit $T$ une sous-variété irréductible de $\mathcal{C}(X)$ contenant $\{C\}=t_{0}$. L'ensemble $T^{\prime}$ (resp. $\left.T^{\prime \prime}\right)$ des $t \in T$ tels que $C_{t}$ soit immergée (resp. T-libre) est un ouvert de Zariski de T. Si $T^{\prime}$ et $T^{\prime \prime}$ sont non-vides, alors le sous-ensemble $T^{*}$ des $t \in T$ pour lesquels $C_{t}$ est immergée et strictement $T$-libre est un ouvert de Zariski non vide de $T$.

Démonstration. - Soit $G^{\prime \prime}:=\left\{g \in G\right.$ tels que $\left.\operatorname{dim}_{g}\left(q^{-1} q(g)\right)>r\right\}$, où $r:=\operatorname{dim}(G)-\operatorname{dim}(X)$. On a $\left(T-T^{\prime \prime}\right)=p\left(G^{\prime \prime}\right)$, d'où la première assertion.

La seconde résulte de ce que $T^{\prime}=(p \circ \tilde{\nu})\left(\widehat{G}^{\prime}\right.$, où

$$
\begin{aligned}
& \widehat{G}^{\prime}:=\{\hat{g} \in \widehat{G} \text { tels que } \widehat{G} \text { est lisse en } \tilde{g}, \\
& \left.\qquad \text { et } \tilde{q}^{-1}(\tilde{q}(\tilde{g})) \text { est réduit et de dimension } r \text { en } \hat{g}\right\} .
\end{aligned}
$$

La troisième résulte de ce que l'ensemble des $\hat{g}$ de $\widehat{G}^{\prime}$ en lesquels $\tilde{q}^{-1}(\tilde{q}(\tilde{g}))$ n'est pas lisse est de codimension au moins deux dans $\widehat{G}^{\prime}$, d'après $[C]$, appendice. 
Proposition 3.5. - Les deux propriétés suivantes sont équivalentes:

1) C est T-libre.

2) Pour toute sous-variété $Y$ de $X$ de codimension au moins 2 dans $X$, l'ensemble des $t \in T$ tels que $\left(C_{t} \cap Y\right)=\emptyset$ est un ouvert de Zariski dense de $T$.

Démonstration.

$1 \Rightarrow 2$, car si $Z$ était une composante irréductible de $q^{-1}(Y)$ telle que $p(Z)=T$, on aurait :

$$
\operatorname{codim}_{G}(Z)=\operatorname{codim}_{X}(Y) \leq 1,
$$

puisque $Z$ rencontre $G^{\prime \prime}$, contrairement à l'hypothèse. Donc $p\left(q^{-1}(Y)\right) \neq T$ et l'ensemble des $t \in T$ tels que $\left(C_{t} \cap Y\right)=\emptyset$ est l'ouvert dense $T / p\left(q^{-1}(Y)\right)$. $2 \Rightarrow 1$, car si $p\left(G^{\prime \prime}\right)=T, q\left(G^{\prime \prime}\right)$ est de codimension au moins deux dans $X$. Or, $G^{\prime \prime}$ est l'ensemble des points en lesquels $q$ n'est pas ouverte et l'on vient de montrer que $p\left(G^{\prime \prime}\right) \neq T$. Donc $q$ est ouverte le long de $p^{-1}(t)$ si $t \in T$ est dans l'ouvert de Zariski non vide $T / p\left(G^{\prime \prime}\right)$.

REMARQUE 3.6. - La notion de liberté peut être définie de manière analogue pour les cycles de dimension quelconque. Lorsque $h^{1}\left(X, O_{X}\right)=0$, cette notion coïncide avec le fait d'appartenir à un système linéaire sans point base, pour des cycles de codimension 1 .

Proposition 3.7 (voir [N]). - Soit $X$ une variété de Fano de dimension $n$. Alors $X$ contient une courbe rationnelle $C$ strictement libre et immergée telle que $c_{1}(X) \cdot C \leq(n+1)$. (En fait, $[\mathrm{N}]$ contient un résultat un peu plus précis.)

\section{Un théorème de confinement}

THÉORÈme 4.1. - Soit $X$ une variété projective lisse de dimension $n$ et $L \in \operatorname{Pic}(X)$, ample sur $X$. Soit $C$ une courbe $T$-libre immergée de $X$ telle que :

$$
d_{L}(C)<\frac{1}{n}
$$

Pour tout $x$ de $X$, il existe une sous-variété projective $V$ de $X$ telle que :

1) $x \in V$.

2) $V \varsubsetneqq X$.

3) Pour tout $t$ de $T$ tel que $C_{t}$ soit strictement $T$-libre et immergée et rencontre $V$, la courbe $C_{t}$ est contenue dans $V$.

$$
\text { TOME } 119-1991-\mathrm{N}^{\circ} 4
$$


Lemme 4.2. - Il existe $m>0$ entier et $D \in|m L|$ tel que $x \in S\left(\frac{n D}{1+n m d}\right), \quad$ avec $: d:=L \cdot C$, où $S\left(\frac{n D}{1+n m d}\right)$ est le lieu de non-intégrabilité de $\left(\frac{n D}{1+n m d}\right)$ (voir $\S 1$ ).

Démonstration. - Soit $\mu>0$ entier tel que $(\mu L-K)$ soit ample, où $K:=K_{X}$ est le fibré canonique de $X$. Alors si $m \geq \mu$, et si $i>0$ :

$$
h^{i}(X, m L)=h^{i}(X,(m-\mu) L+(\mu L-K)+K)=0 .
$$

Donc :

$$
\chi(X, m L)=h^{0}(X, m L)=\frac{m^{n} L^{n}}{n !}(1+\varepsilon(m)),
$$

où $\varepsilon(m)$ tend vers 0 lorsque $m$ tend vers $+\infty$. D'autre part,

$$
\begin{aligned}
h^{0}\left(X,\left(O_{X, x} / \mathcal{M}_{X, x}^{n \cdot m \cdot d+2}\right) \otimes_{\mathbb{C}} L\right) \\
\quad=\left(\begin{array}{c}
n+2+n \cdot m \cdot d \\
n
\end{array}\right)=\frac{(n \cdot m \cdot d)^{n}}{n !}(1+\eta(m))
\end{aligned}
$$

où $\eta(m) \rightarrow 0$ quand $m \rightarrow+\infty$ si $d:=(L \cdot C)$ et où $\mathcal{M}_{x, X}$ est l'idéal maximal de $\mathcal{O}_{X, x}$ en $x$.

L'inégalité $d_{L}(C)<1 / n$ implique donc que l'application naturelle d'évaluation à l'ordre $(n \cdot m \cdot d)$ en $x$ :

$$
\text { ev : } H^{0}(X, m L) \longrightarrow H^{0}\left(X,\left(\mathcal{O}_{X, x} / \mathcal{M}_{X, x}^{n \cdot m \cdot d+2}\right) \otimes L\right)
$$

n'est pas injective si $m$ est assez grand. Donc $|m L|$ contient un membre $D$ s'annulant à un ordre strictement supérieur à $(n \cdot m \cdot d)$ en $x$. Ceci montre que $x$ appartient à $S\left(\frac{n D}{1+n m d}\right)$.

On notera que $\left(\frac{n D}{1+n m d}\right) \cdot C<1$.

Pour conclure la démonstration du THÉORÈme 4.1, on pose :

$$
V:=S\left(\frac{n D}{1+n m d}\right) \text {. }
$$

Soit maintenant $t \in T^{*}$ (c'est-à-dire $C_{t}$ est strictement $T$-libre et immergée). Soit $\tilde{p}:=p \circ \tilde{\nu}: \widehat{G} \rightarrow T$ et soit $\tilde{q}: q \circ \tilde{\nu}: \widehat{G} \rightarrow X:$ ce sont des submersions au voisinage de $\widehat{G}_{t}:=(p \circ \tilde{\nu})^{-1}(t)$. On suppose qu'il existe $x_{0} \in\left(C_{t} \cap V\right)$. Il existe donc une immersion $\varphi: \Delta^{n-1} \rightarrow T$ telle que $\varphi(0)=t$, où $\Delta^{n-1}$ est un polydisque de $\mathbb{C}^{n-1}$ centré en 0 , tel que, si $q_{\Delta}: G_{\Delta} \rightarrow X$ et $p_{\Delta}: G_{\Delta} \rightarrow \Delta^{n-1}$ sont déduits de $\tilde{q}$ et $\tilde{p}$ par le changement de base $\varphi: \Delta^{n-1} \rightarrow T$, alors $q_{\Delta}$ est un isomorphisme local au voisinage du point $\left(0, x_{0}\right)$. Il résulte alors du THÉORÈme 2.1 que $(0) \times C_{t}$ est contenu dans $q_{\Delta}^{*}(V)=S\left(q_{\Delta}^{*}\left(\frac{n D}{1+n m d}\right)\right)$. 


\section{Un diviseur non ample}

5.1 Notations. - On utilise les notations introduites en 3.1. On suppose que $\left(C_{t}\right)_{t \in T}$ est une famille de courbes de $X$ et que

$T^{*}:=\left\{t \in T\right.$ tels que $C_{t}$ est strictement $T$-libre et immergée $\}$

est non vide. C'est donc un ouvert de Zariski dense de $T$.

DÉfinition 5.2. - Soit $V \neq \emptyset$ une sous-variété projective de $X$, distincte de $X$. On dit que $V$ est $T$-saturée si, pour tout $t$ de $T^{*}$ tel que $C_{t}$ rencontre $V$, la courbe $C_{t}$ est contenue dans $V$.

On note $\mathcal{S}(T)$ l'ensemble des sous-variétés $T$-saturées de $X$ si $x \in X$; on note $\mathcal{S}(T)_{x}$ le sous-ensemble des $V$ de $\mathcal{S}(T)$ qui contiennent $x$.

ThÉoRÈme 5.3. - Soit $X$ une variété projective lisse et connexe de dimension $n$, soit $C$ une courbe immergée strictement $T$-libre de $X$. On suppose que, pour tout $x$ de $X$, l'ensemble $\mathcal{S}(T)_{x}$ est non vide. Il existe alors un diviseur effectif $E$ de $X$ tel que $E \cdot C=0$.

LEMME 5.4 .

i) L'ensemble $\mathcal{S}(T)$ est stable par intersection.

ii) Si $V_{1}$ et $V_{2}$ sont deux sous-variétés de $X$, si aucune ne contient l'autre et si $\left(V_{1} \cup V_{2}\right) \in \mathcal{S}(T)$, alors $V_{1}$ et $V_{2}$ sont dans $\mathcal{S}(T)$.

\section{Démonstration.}

i) est évident, car si $V_{\sigma} \in \mathcal{S}(T)$ pour tout $\sigma \in \Sigma$, si $t \in T^{*}$ et si $\left(C_{t} \cap V\right) \neq \emptyset$, où $V=\left(\bigcap_{\sigma \in \Sigma} V_{\sigma}\right)$, alors $C_{t}$ est contenue dans $V_{\sigma}$ pour tout $\sigma$ de $\Sigma$. Donc $C_{t} \subset V$.

ii) Soit $t \in T^{*}$; si $\left(C_{t} \cap V_{1}\right)$ est non vide et non contenu dans $V_{2}$, alors $C_{t} \subset V_{1}$, puisque $C_{t}$ est irréductible. Sinon, soit $s \in\left(C_{t} \cap V_{1} \cap V_{2}\right)$. Il existe donc une application analytique $\alpha: \Delta \rightarrow T^{*}$ telle que $\alpha(0)=t$ et telle que $\left(C_{\alpha(\xi)} \cap V_{1}\right)$ ne soit pas contenu dans $V_{2}$ si $\xi \neq 0$, où $\Delta$ est le disque unité de $\mathbb{C}$. L'existence de $\alpha$ provient de ce que $C_{t}$ est $T$-libre.

On a donc $C_{\alpha(\xi)} \subset V_{1}$ pour $\xi \neq 0$, donc aussi pour $\xi=0$. Donc $V_{1} \in \mathcal{S}(T)$.

Corollaire 5.5. - Pour tout $x$ de $X$, l'ensemble $\mathcal{S}(T)_{x}$ admet un élément minimum pour l'inclusion, noté $V_{x}$ et $V_{x}$ est irréductible.

Démonstration. - La première assertion résulte immédiatement de 5.4.i) et la seconde de 5.4.ii) puisque

$$
\begin{gathered}
\left(V_{1} \cup V_{2}\right) \in \mathcal{S}(T)_{x} \text { et } x \in\left(V_{1}-V_{2}\right) \Rightarrow V_{1} \in \mathcal{S}(T)_{x} \\
x \in\left(V_{1} \cap V_{2}\right) \Rightarrow\left(V_{1} \cap V_{2}\right) \in \mathcal{S}(T)_{x}
\end{gathered}
$$

TOME $119-1991-\mathrm{N}^{\circ} 4$ 
Notation : soit $X^{*}$ l'ouvert de Zariski dense de $X$ constitué des $x$ tels que $\overline{T_{x}^{*}}=T_{x}$, où :

$$
T_{x}=p\left(q^{-1}(x)\right), \quad T_{x}^{*}=\left(T_{x} \cap T^{*}\right),
$$

et où $\overline{T_{x}^{*}}$ est l'adhérence de $T_{x}^{*}$ dans $T$.

On se propose maintenant de fournir une autre description de $V_{x}$, pour $x$ dans $X^{*}$.

Notation 5.7. - Pour $U$ contenu dans $X^{*}$, on note $T(U)$ le sous ensemble de $X^{*}$ défini par :

$$
T(U)=\left\{\overline{q\left(p^{-1}\left(p\left(q^{-1}(U)\right)\right)\right)} \cap X^{*}\right\} .
$$

Donc $T(U) \supset U$.

On remarque que $T(U)=q\left(p^{-1}\left(\overline{p\left(q^{-1}(u)\right)}\right)\right) \cap X^{*}$ et que c'est l'adhérence dans $X^{*}$ des points de $X^{*}$ par lesquels passe une courbe $C_{t}$, $t \in T^{*}$, qui rencontre $U$.

\section{LEMME 5.8 .}

i) Si $U$ est un fermé de Zariski de $X^{*}, T(U)$ est un fermé de Zariski de $X^{*}$. (Ici la topologie de Zariski est prise au sens algébrique.)

ii) Si $V \in \mathcal{S}(T)$ et si $U \subset\left(V \cap X^{*}\right)$, alors $T(U) \subset\left(V \cap X^{*}\right)$.

iii) Si $U$ est un fermé de Zariski de $X^{*}$, si $U^{0}$ est une composante irréductible de $U$ qui est aussi une composante irréductible de $T(U)$, alors $\bar{U} \in \mathcal{S}(T)$.

Démonstration. - L'assertion i) résulte immédiatement de la définition, puisque $q\left(p^{-1}\left(\overline{p\left(q^{-1}(U)\right)}\right)\right)$ est Zariski fermé dans $X$. L'assertion ii) résulte immédiatement des définitions.

Dans la situation de iii), soit $t \in T^{*}$ tel que $\left(C_{t} \cap U^{0}\right)$ soit non vide. Si $\left(C_{t} \cap U^{0}\right)$ n'est pas contenu dans $\overline{\left(T(U)-U^{0}\right)}$, alors $C_{t}$ est contenue dans $U^{0}$, puisque $C_{t}$ est contenue dans $\overline{T(U)}$ par définition.

Sinon, la démonstration de 5.4.ii) permet de se ramener à ce cas, en y remplaçant $\left(V_{1} \cup V_{2}\right) \operatorname{par} \overline{T(U)}$ et $V_{1} \operatorname{par} \overline{U^{0}}$.

Notation 5.9. - Pour $x$ dans $X^{*}$, on note :

$$
T^{0}(x):=\{x\} \quad \text { et } \quad T^{n+1}(x):=T\left(T^{n}(x)\right) \text { pour } n \geq 0 .
$$

On définit ainsi une suite croissante de fermés de Zariski de $X^{*}$. On note $T^{\infty}(x)$ la réunion des $T^{n}(x), n \geq 0$. 
Lemme 5.10. $-T^{\infty}(x)=\left(V_{x} \cap X^{*}\right)$ pour tout $x$ de $X^{*}$ et il existe un entier $N(x)$ tel que $T^{\infty}(x)=T^{N(x)}(x)$.

Démonstration. - On vérifie immédiatement par récurrence sur $N$ que $T^{N}(x)$ est contenu dans $V$ pour tout $V \in \mathcal{S}(T)_{x}$, ceci à l'aide de 5.8.iii). D'où l'inclusion $T^{\infty}(x) \subset\left[V_{x} \cap X^{*}\right]$. On montre l'inclusion en sens inverse.

Soit, pour tout $N, d_{N}$ la dimension maximum des composantes irréductibles de $T^{N}(x)$ qui contiennent $x: d_{N}$ est une suite bornée (par $n$ ) d'entiers; donc stationnaire.

Soit $N_{0}$ tel que $d_{N_{0}}=\left(\sup _{N \geq 0} d_{N}\right)$, et soit $R^{*}$ une composante irréductible de $T^{N_{0}}(x)$ contenant $x$ et telle que $\operatorname{dim}\left(R^{*}\right)=d_{N_{0}}$. Alors $R^{*}$ est encore une composante irréductible de $T^{n_{0}+1}(x)=T\left(T^{N_{0}}(x)\right)$ qui contient $x$. Donc $R:=\overline{R^{*}}$ appartient à $\mathcal{S}(T)_{x}$ et l'on a :

$$
T^{\infty}(x) \supset T^{N_{0}}(x) \supset R^{*}=\left(R \cap X^{*}\right) \supset\left(V_{x} \cap X^{*}\right)
$$

On en déduit l'égalité :

$$
T^{N_{0}}(x)=T^{\infty}(x)=\left[V_{x} \cap X^{*}\right]
$$

Proposition 5.11. - Il existe un ouvert de Zariski non vide $X^{* *}$ de $X$, contenu dans $X^{*}$ tel que si $x$ et $y$ sont dans $X^{* *}$, et si $V_{x}^{* *}:=\left(V_{x} \cap X^{* *}\right)$ rencontre $V_{y}^{* *}$, alors $V_{x}=V_{y}$ et il existe une variété quasi-projective $W^{*}$ et un morphisme algébrique

$$
\mu: X^{* *} \longrightarrow W^{*} \text { tels que } \mu^{-1} \mu(x)=V_{x}^{* *} \text { pour tout } x \text { de } X^{* *} \text {. }
$$

La démonstration de ce résultat va nécessiter quelques préliminaires.

Notations 5.12. - Pour $U \subset X^{*}$, on notera :

$$
T^{*}(A):=q\left(p^{-1}\left(T^{*} \cap p\left(q^{-1}(A)\right)\right)\right) \cap X^{*}
$$

et par récurrence sur $N \geq 0$ entier

$$
T^{*(0)}(A):=A, \quad T^{*(N)}(A):=T^{*}\left(T^{*(N-1)}(A)\right) \quad \text { si } \quad N \geq 1 .
$$

Lemme 5.13. - Pour tout $A$ de $X^{*}$, on a

$$
T\left(\bar{A} \cap X^{*}\right)=\overline{T^{*}(A)} \cap X^{*},
$$

où la barre désigne l'adhérence dans $X$ pour la topologie métrique.

$$
\text { TOME } 119-1991-\mathrm{N}^{\circ} 4
$$


Démonstration. - Il suffit d'établir l'inclusion :

$$
T\left(\bar{A} \cap X^{*}\right) \subset \overline{T^{*}(A)} \cap X^{*},
$$

puisque l'inclusion opposée est évidente. Or, elle résulte de ce que

$$
\left(\left(q^{-1}\left(\bar{A} \cap X^{*}\right)\right) \cap T^{*}\right) \quad \text { est dense dans } p\left(q^{-1}\left(\bar{A} \cap X^{*}\right)\right),
$$

par définition de $X^{*}$, et de ce que

$$
q^{-1}\left(\bar{A} \cap X^{*}\right) \subset \overline{q^{-1}(A)},
$$

puisque

$$
q^{-1}(x)=\lim _{x^{\prime} \rightarrow x}\left(q^{-1}\left(x^{\prime}\right)\right) \quad \text { si } x \text { est dans } X^{*} ;
$$

puisque le morphisme $q$ est à fibres équidimensionnelles au-dessus de $X^{*}$; la limite ci-dessus peut-être prise au sens de la topologie métrique de la variété de Chow de $(T \times X)$.

Lemme 5.14. - Si A est fermé dans $X^{*}$, alors :

i) $T(A)=\overline{T^{*}(A)} \cap X^{*}$;

ii) $T^{N}(A)=\overline{T^{*(N)}(A)} \cap X^{*}$;

iii) $V_{x}^{*}=\overline{T^{*(N(x))}(x)} \cap X^{*}$.

\section{Démonstration.}

i) est un cas particulier de 5.12. On obtient ii) par récurrence sur $N$ en appliquant 5.12 à $B=T^{*(N)}(A)$. On a alors

$$
\bar{B} \cap X^{*}=T^{N}(A)
$$

par l'hypothèse de récurrence. Donc :

$$
T^{N+1}(A)=T\left(\bar{B} \cap X^{*}\right)=\overline{T^{*}(B)} \cap X^{*}=\left(\overline{T^{*(N+1)}(A)}\right) \cap X^{*} .
$$

On obtient iii) en appliquant ii) à l'égalité 5.10.

Notation 5.15. - Soit $N \geq 0$ entier. On note $R^{* N} \subset X^{*} \times X^{*}$ le graphe de la relation binaire $(x, y) \in R^{* N}$ si $y \in T^{* N}(x)$. On remarquera que cette relation est symétrique (c'est-à-dire $(y, x) \in R^{* N}$ si $\left.(x, y) \in R^{* N}\right)$. On notera $q_{N}$ (resp. $q_{N}^{\prime}$ ) la restriction à $R^{* N}$ de la projection naturelle de $X^{*} \times X^{*}$ sur son premier (respectivement second) facteur. Soit $\bar{q}_{N}$ (resp. $\bar{q}_{N}^{\prime}$ ) la restriction de cette projection à $R^{N}:=\overline{R^{* N}}$, adhérence de $R^{* N}$ dans $X \times X$. 
LEMME 5.16. - Il existe un ouvert de Zariski dense $X^{* *}$ de $X$, contenu dans $X^{*}$, et un entier $N_{0}$ tels que pour tout $x$ de $X^{* *}, \overline{q_{N_{0}}^{-1}(x)}=$ $\left(\bar{q}_{N_{0}}\right)^{-1}(x)=V_{x}$, et de dimension constante $d$.

Démonstration. - Pour tout $N$, l'ensemble des $x$ de $X^{*}$ pour lesquels $\overline{q_{N}^{-1}(x)}=\left(\bar{q}_{N}\right)^{-1}(x)$ contient un ouvert de Zariski $U_{N}^{*}$ de $X^{*}$. Soit $N_{0}$ un entier tel que $R^{* N_{0}}$ contienne une composante irréductible contenant la diagonale $D^{*}$ de $X^{*} \times X^{*}$, et de dimension maximum $d^{+}$parmi toutes les composantes irréductibles de $R^{* N}$ possédant cette propriété, pour $N \geq N_{0}$.

Soit $x$ dans $X^{* *}:=U_{N_{0}}^{*}$. D'après le LEMmE $5.14,\left(\bar{q}_{N}\right)^{-1}(x)=V_{x}$, et d'après la définition de $X^{* *}, \overline{\left(q_{N}^{-1}(x)\right)}=V_{x}$ est de dimension minimum $d=\left(d^{+}-\operatorname{dim}(X)\right)$, quitte à restreindre $X^{* *}$ à l'ouvert au-dessus duquel la dimension de $\overline{q_{N}^{-1}(x)}$ est constante.

On peut maintenant démontrer 5.11 ; si $z \in\left(V_{x}^{* *} \cap V_{y}^{* *}\right)$, on a $V_{z} \subset\left(V_{x} \cap V_{y}\right)$, puisque cette intersection est dans $\mathcal{S}(T)_{z}$ d'après 5.4.i). Mais chacune des variétés $V_{x}, V_{y}$ et $V_{z}$ est irréductible par 5.4. ii), et de dimension $d$, par 5.16. Donc $V_{x}=V_{y}=V_{z}$. D'où i).

Puisque $X^{* *}$ est lisse, donc normale, et que la dimension $d$ des fibres de $R^{N_{0}}$ est constante au-dessus de $X^{*},\left(\bar{q}_{N}\right)^{-1}\left(X^{* *}\right):=R^{* *}$ est le graphe d'incidence d'une famille algébrique de cycles de $X$ paramétrée par $X^{* *}$, et associée à l'application algébrique $\mu: X^{* *} \rightarrow W^{* *}$ définie par $\mu(x)=\nu(x) \cdot V_{x}$, où $\nu(x) \in \mathbb{N}^{*}$ est une multiplicité convenable, génériquement égale à un, et où $W^{* *}$ est un ouvert de Zariski dense d'une composante irréductible $W$ de la variété de Chow $C_{d}(X)$ des cycles algébriques de dimension $d$ de $X$. Ceci établit donc 5.11 .

Le Théorème 5.3 résulte alors du LEMme 5.17 suivant :

Lemme 5.17. - Soit $X$ une variété projective irréductible de dimension $n$; soit $U$ un ouvert de Zariski dense de $X$, et soit $\varphi: U \rightarrow V$ un morphisme algébrique à valeurs dans une variété quasi-projective $V$. Soit $C$ une courbe immergée strictement $T$-libre de $X$ rencontrant $U$. On suppose que, pour tout $x$ de $U, \overline{\varphi^{-1}(\varphi(x))}$ est dans $\mathcal{S}(T)$ (au sens de la définition 5.2). Il existe alors un diviseur effectif $E$ de $X$ tel que $E \cdot C=0$.

Démonstration. - Soit $\Delta$ un diviseur effectif de $V$ ne passant pas par $\varphi(C \cap U)$; un tel diviseur existe, puisque $\varphi(C \cap U)=\varphi(x)$ si $x \in(C \cap U)$. Soit $E:=\overline{\varphi^{*}(\Delta)}$; alors $Y:=(E \cap(X / U))$ est de codimension au moins 2 dans $X$.

Quitte à remplacer $C=C_{t_{0}}$ par une courbe voisine $C_{t}$, c'est-à-dire paramétrée par un point voisin $t$ de $t_{0}$ dans $T$, on peut donc supposer

TOME $119-1991-\mathrm{N}^{\circ} 4$ 
que $C_{t} \cap Y=\emptyset$. Mais alors $C_{t} \cap E=\emptyset$, puisque $\varphi\left(C_{t} \cap U\right)$ est un point voisin de $x$ et ne rencontre donc pas $\Delta$.

\section{Le résultat principal}

THÉorème 6.1. - Soit $X$ une variété projective et connexe de dimension $n$; soit $L \in \operatorname{Pic}(X)$ ample; soit $C$ une courbe libre et immergée de $X$ telle que $d_{L}(C)<1 / n$. Alors $X$ contient un diviseur effectif $E$ tel que $E \cdot C=0$. En particulier $\rho(X) \geq 2$.

C'est une conséquence immédiate de 5.3 et 4.1 .

Corollaire 6.2. - Si $X$ et L sont comme en 6.1. Si $\rho(X)=1$, alors pour toute courbe libre et immergée $C$, on a :

$$
d_{L}(C) \geq \frac{1}{n} .
$$

Corollaire 6.3. - Si $X$ est une variété de Fano avec $b_{2}(X)=1$, on a $c_{1}(X)^{n} \leq(n \cdot d(X))^{n} \leq(n \cdot(n+1))^{n}$, où $d(X)$ est le minimum des valeurs $c_{1}(X) \cdot C$, pour $C$ rationnelle libre et immergée de $X$. (La seconde inégalité résulte de 3.7 .)

\section{BIBLIOGRAPHIE}

[C] Campana (F.). - Coréduction algébrique d'un espace analytique faiblement Kählérien compact, Invent. Math., t. 63, 1981, p. 187-223.

[D] Demailly (J.-P.). - Transcendental proof of a generalized Kawamata-Viehweg vanishing theorem. - Preprint, 1988.

[K] KodAiRA (K.). - A theorem of completeness of characteristic systems for analytic families of compact submanifolds of complex manifolds, Ann. Math., t. 75, 1962, p. 146-162.

[K-M] Kollar (J.) and Matsusaka (T.). - Riemann-Roch type inequalities, Amer. J. Math., t. 105, 1983, p. 229-252.

[N] Nadel A.). - A finiteness theorem for Fano 4. Folds. - Preliminary version.

[N'] NADEL (A.). - The boundedness of degree of Fano Varieties with Picard number one. - Preprint MIT, 1990.

[T] TsujI (H.). - Boundness of the degree of Fano manifolds with $b_{2}=1$, Preprint 1990. 